\title{
Proof of the Seymour Conjecture for Large Graphs
}

\author{
János Komlós ${ }^{1}$, Gábor N. Sárközy ${ }^{2}$, and Endre Szemerédi ${ }^{3}$ \\ ${ }^{1}$ Department of Mathematics, Rutgers University, New Brunswick, NJ 08930, USA \\ komlos@math.rutgers.edu \\ ${ }^{2}$ Computer Science Department, Worcester Polytechnic Institute, Worcester, MA 01609, USA \\ gsarkozy@cs.wpi.edu \\ ${ }^{3}$ Computer Science Department, Rutgers University, New Brunswick, NJ 08930, USA \\ szemered@cs.rutgers.edu
}

Received December 5, 1997

AMS Subject Classification: 05C45

\begin{abstract}
Paul Seymour conjectured that any graph $G$ of order $n$ and minimum degree of at least $\frac{k}{k+1} n$ contains the $k$ th power of a Hamiltonian cycle. Here, we prove this conjecture for sufficiently large $n$.
\end{abstract}

Keywords: dense graphs, powers of Hamiltonian cycles

\section{Introduction}

\subsection{Notations and Definitions}

For basic graph concepts see the monograph of Bollobás [1]. + will sometimes be used for disjoint union of sets. $V(G)$ and $E(G)$ denote the vertex-set and the edge-set of the graph $G$. $(A, B, E)$ denotes a bipartite graph $G=(V, E)$, where $V=A+B$, and $E \subset A \times B$. For a graph $G$ and a subset $U$ of its vertices, $\left.G\right|_{U}$ is the restriction to $U$ of $G$. $N(v)$ is the set of neighbors of $v \in V$. Hence, $|N(v)|=\operatorname{deg}(v)=\operatorname{deg}_{G}(v)$, the degree of $v . \delta(G)$ stands for the minimum and $\Delta(G)$ the maximum degree in $G . v_{i}(G)$ denotes the size of a maximum set of vertex disjoint paths of length $i$ (counting edges) in $G$. (Thus, $v_{1}(G)=v(G)$ is the size of a maximum matching.) For $A \subset V(G)$, we write

$$
N(A)=\bigcap_{v \in A} N(v)
$$

the set of common neighbors. $N(x, y, z, \ldots)$ is shor for $N(\{x, y, z, \ldots\})$. When $A, B$ are subsets of $V(G)$, we denote by $e(A, B)$ the number of edges of $G$ with one endpoint in 
$A$ and the other in $B$. In particular, we write $\operatorname{deg}(v, U)=e(\{v\}, U)$ for the number of edges from $v$ to $U$. For non-empty $A$ and $B$,

$$
d(A, B)=\frac{e(A, B)}{|A||B|}
$$

is the density of the graph between $A$ and $B$. In particular, we write $d(A)=d(A, A)=$ $2\left|E\left(\left.G\right|_{A}\right)\right| /|A|^{2}$.

Definition 1.1. The bipartite graph $G=(A, B, E)$ is $\varepsilon$-regular if

$$
X \subset A, Y \subset B,|X|>\varepsilon|A|,|Y|>\varepsilon|B| \text { imply }|d(X, Y)-d(A, B)|<\varepsilon,
$$

otherwise, it is E-irregular.

We will often simply say that "the pair $(A, B)$ is $\varepsilon$-regular" with the graph $G$ implicit.

Definition 1.2. $(A, B)$ is $(\varepsilon, \delta)$-super-regular if it is $\varepsilon$-regular and

$$
\operatorname{deg}(a)>\delta|B| \forall a \in A, \quad \operatorname{deg}(b)>\delta|A| \forall b \in B .
$$

\subsection{Powers of Cycles}

The $k$ th power of a graph $G$ is the graph obtained from $G$ by joining every pair of vertices with a distance of at most $k$ in $G$.

Let $G$ be a graph on $n \geq 3$ vertices. A classical result of Dirac [2] (see also [1]) asserts that if $\delta(G) \geq n / 2$, then $G$ contains a Hamiltonian cycle. As a natural generalization of Dirac's theorem, Pósa conjectured the following in 1962.

Conjecture 1.3.(Pósa) Let $G$ be a graph on $n$ vertices. If $\delta(G) \geq \frac{2}{3} n$, then $G$ contains the square of a Hamiltonian cycle.

Later, in 1974, Seymour [16] generalized this conjecture.

Conjecture 1.4. [16] Let $G$ be a graph on $n$ vertices. If $\delta(G) \geq \frac{k}{k+1} n$, then $G$ contains the kth power of a Hamiltonian cycle.

Seymour indicated the difficulty of the conjecture by observing that the truth of this conjecture would imply the notoriously difficult Hajnal-Szemerédi theorem [10] (see below).

The problem received significant attention lately. In the direction of Conjecture 1.3 , Jacobson (unpublished) first showed that if $\delta(G) \geq \frac{5}{6} n$, then the conclusion of the conjecture holds. Faudree, et al. [8] confirmed the conclusion that

$$
\delta(G) \geq\left(\frac{3}{4}+\varepsilon\right) n+C(\varepsilon) .
$$

Later the same authors improved this to $\delta(G) \geq \frac{3}{4} n$. By using a result in [9], Häggkvist (unpublished) gave a very simple proof for the case $\delta(G) \geq \frac{3}{4} n+1$ and $n \equiv 0(\bmod$ 4). Fan and Häggkvist [3] lowered the bound to $\delta(G) \geq \frac{5}{7} n$. Fan and Kierstead [4] 
improved this further to $\delta(G) \geq \frac{17 n+9}{24}$, and Faudree, Gould and Jacobson [7] to $\delta(G) \geq$ $\frac{7}{10} n$. Later, Fan and Kierstead [5] improved the condition to the almost optimal $\delta(G) \geq$ $\left(\frac{2}{3}+\varepsilon\right) n+C(\varepsilon)$. They also proved [6] that the same holds with $\varepsilon=C=0$ if one only requires the square of a Hamiltonian path. Finally, in [13] we proved the conjecture for sufficiently large $n$.

For Conjecture 1.4, in [8], it is proved that for any $\varepsilon>0$ and positive integer $k$, there is a $C$ such that, if an $n$-graph $G$ satisfies

$$
\delta(G) \geq\left(\frac{2 k-1}{2 k}+\varepsilon\right) n+C,
$$

then $G$ contains the $k$ th power of a Hamiltonian cycle.

In [14], we proved the following improvement of this result. For any $\varepsilon>0$ and positive integer $k$, there is an $N(\varepsilon, k)$ such that if $G$ has order $n$ with $n \geq N(\varepsilon, k)$ and

$$
\delta(G) \geq\left(\frac{k}{k+1}+\varepsilon\right) n
$$

then $G$ contains the $k$ th power of a Hamiltonian cycle.

Here, the purpose is to prove Conjecture 1.4 for any fixed $k$ and sufficiently large $n$. More precisely:

Theorem 1.5. For any positive integer $k$, there is an $N(k)$ such that, if $G$ has order $n$ with $n \geq N(k)$ and

$$
\delta(G) \geq \frac{k}{k+1} n,
$$

then $G$ contains the kth power of a Hamiltonian cycle.

\section{The Main Tools}

In the proof, the Regularity Lemma of the third author plays a central role. Here we will use the following variation of the lemma.

Lemma 2.1.(Regularity Lemma - degree form) For every $\varepsilon>0$, there is an $M=M(\varepsilon)$ such that, if $G=(V, E)$ is any graph and $d \in[0,1]$ is any real number, then there is a partition of the vertex-set $V$ into $l+1$ sets (so-called clusters) $V_{0}, V_{1}, \ldots, V_{l}$, and there is a subgraph $G^{\prime}=\left(V, E^{\prime}\right)$ with the following properties:

- $l \leq M$,

- $\left|V_{0}\right| \leq \varepsilon|V|$,

- all clusters $V_{i}, i \geq 1$ are of the same size $L \leq\lceil\varepsilon|V|\rceil$.

- $\operatorname{deg}_{G^{\prime}}(v)>\operatorname{deg}_{G}(v)-(d+\varepsilon)|V|$ for all $v \in V$,

- $\left.G^{\prime}\right|_{V_{i}}=\emptyset\left(V_{i}\right.$ are independent in $\left.G^{\prime}\right)$,

- all pairs $\left.G^{\prime}\right|_{V_{i} \times V_{j}}, 1 \leq i<j \leq$ l are $\varepsilon$-regular, each with a density 0 or exceeding $d$. 
This form can easily be obtained by applying the original Regularity Lemma (with a smaller value of $\varepsilon$ ), adding to the exceptional set $V_{0}$ all clusters incident to many irregular pairs, and then deleting all edges between any other clusters where the edges either do not form a regular pair or they do but with a density of at most $d$.

The other main tool is a coloring theorem of Hajnal and Szemerédi which states that every graph with $n$ vertices and maximum degree $\Delta(G) \leq k$ is $(k+1)$-colorable with all color classes of size $\lfloor n /(k+1)\rfloor$ or $\lceil n /(k+1)\rceil$. We have already pointed out the close connection between Seymour's problem and the Hajnal-Szemerédi theorem, namely, the truth of Conjecture 1.4 would imply the latter theorem. We use the theorem in the following complementary form.

Lemma 2.2.[10] Let $G$ be a graph on $n=s(k+1)$ vertices. If $\delta(G) \geq \frac{k}{k+1} n$, then $G$ contains $s$ vertex-disjoint cliques of order $k+1$.

In fact, we are going to use the following easy consequence of this lemma.

Lemma 2.3. Let $G$ be a graph on $n$ vertices. If $\delta(G) \geq \frac{k}{k+1} n-x$ for some natural number $x$, then apart from at most $k(k+1) x+k^{2}$ exceptional vertices, $V(G)$ can be covered by vertex-disjoint cliques of order $k+1$.

Indeed, add $(k+1) x$ extra vertices to $G$ and possibly a few $(\leq k)$ more to achieve that the new number of vertices is divisible by $k+1$. Connect the new vertices to all other vertices. Denote the resulting graph by $\widetilde{G}$ and the new number of vertices by $\tilde{n}$. It is easy to see that $\delta(\widetilde{G}) \geq \frac{k}{k+1} \tilde{n}$, therefore, using Lemma 2.2 , we can cover $\widetilde{G}$ by vertexdisjoint cliques of order $k+1$. The number of vertices in $V(G)$ which are in cliques containing at least one extra vertex $(\in V(\widetilde{G}) \backslash V(G))$ is at most $k(k+1) x+k^{2}$.

We also use the Blow-up Lemma (see $[12,15]$ ).

Lemma 2.4. Given a graph $R$ of order $r$ and positive parameters $\delta, \Delta$, there exists an $\varepsilon>0$ such that the following holds. Let $N$ be an arbitrary positive integer, and let $u$ s replace the vertices of $R$ with pairwise disjoint $N$-sets $V_{1}, V_{2}, \ldots, V_{r}$ (blowing up). We construct two graphs on the same vertex-set $V=\bigcup V_{i}$. The graph $R(N)$ is obtained by replacing all edges of $R$ with copies of the complete bipartite graph $K_{N, N}$, and a sparser graph $G$ is constructed by replacing the edges of $R$ with some $(\varepsilon, \delta)$-super-regular pairs. If a graph $H$ with $\Delta(H) \leq \Delta$ is embeddable into $R(N)$, then it is already embeddable into $G$.

When using the Blow-up Lemma, we typically need the following strengthened version: Given $c>0$, there are positive functions $\varepsilon=\varepsilon(\delta, \Delta, r, c)$ and $\alpha=\alpha(\delta, \Delta, r, c)$ such that the Blow-up Lemma remains true if, for every $i$, there are certain vertices $x$ to be embedded into $V_{i}$ whose images are a priori restricted to certain sets $C_{x} \subset V_{i}$ provided that

(i) each $C_{x}$ within a $V_{i}$ is of the size at least $c\left|V_{i}\right|$,

(ii) the number of such restrictions within a $V_{i}$ is not more than $\alpha\left|V_{i}\right|$.

Finally, we are going to use the following simple facts (see $[1,13]$ ):

Lemma 2.5. In a graph $G$ on $n$ vertices, we have

$$
v(G) \geq \max \left\{\delta(G), \delta(G) \frac{n}{4 \Delta(G)}\right\} \text { and } v_{2}(G) \geq(\delta(G)-1) \frac{n}{6 \Delta(G)} .
$$




\section{Outline of the Proof}

In a series of papers [11-15] we have developed a general method based on the Regularity Lemma and the Blow-up Lemma for embedding problems in dense graphs. In this paper we use this method again, so the proof follows a similar rough outline as the proof in [13] for example. However, several new ideas are needed.

We will assume throughout the paper that $n$ is sufficiently large. Furthermore, we may assume that $k \geq 3$, since for $k=2$, we proved Theorem 1.5 in [13] and, for $k=1$, it is just Dirac's theorem. We will use the following main parameters:

$$
\varepsilon \ll d \ll \alpha \ll 1,
$$

where $a \ll b$ means that $a$ is sufficiently small compared to $b$. For simplicity, we do not compute the actual dependencies, although it could be done.

We apply Lemma 2.1 for $G$ with $\varepsilon$ and $d$ as in (3.1). We get a partition of $V(G)$ into clusters $V_{0}, V_{1}, \ldots, V_{l}$. We define the following reduced graph $G_{r}$ : The vertices of $G_{r}$ are the clusters $V_{i}, i \geq 1$, and we have an edge between two clusters if they form an $\varepsilon$-regular pair in $G^{\prime}$ with density exceeding $d$. Since in $G^{\prime}, \delta\left(G^{\prime}\right) \geq\left(\frac{k}{k+1}-(d+\varepsilon)\right) n$, an easy calculation shows that in $G_{r}$, we have

$$
\delta\left(G_{r}\right) \geq\left(\frac{k}{k+1}-3 d\right) l .
$$

Let us apply Lemma 2.3 for $G_{r}$ to obtain a covering of most of the vertices in $G_{r}$ by vertex disjoint cliques of size $k+1$. More precisely, we can cover the vertices of $G_{r}$ apart from an exceptional set of size at most $3 k(k+1) d l+k^{2} \leq 4 k(k+1) d l$. Let us put the vertices of these exceptional clusters into the exceptional set $V_{0}$. For simplicity, $V_{0}$ still denotes the resulting set. Then

$$
\left|V_{0}\right| \leq 4 k(k+1) d l L+\varepsilon n \leq 5 k(k+1) d n .
$$

In the proof first we assume until Sec. 7 that the following extremal condition does not hold for our graph $G$ :

Extremal Condition $(E C)$ : There exists an $A \subset V(G)$ such that

- $|A|=\left\lfloor\frac{n}{k+1}\right\rfloor$ and

- $d(A)<\alpha$.

We show later in Sec. 7 that if this condition does hold, then we can find the $k$ th power of the Hamiltonian cycle. Firstly, in the next section, we show that under the assumption that $\mathrm{EC}$ does not hold, we can slightly modify the clique covering; we can achieve that a constant proportion of the cliques are $(k+2)$-cliques and the rest are $(k+1)$-cliques. This new idea will significantly simplify the adjustment procedure in Sec. 6 . These cliques will be denoted by $K_{1}, K_{2}, \ldots, K_{s}$.

In each clique $K_{i}$, we take an arbitrary ordering of the $k+1$ (or $\left.k+2\right)$ clusters and we denote the clusters in this order by $V_{1}^{i}, V_{2}^{i}, \ldots, V_{k+1}^{i}\left(, V_{k+2}^{i}\right)$. We think of this sequence as a cycle of length $k+1$ (or $k+2)$, where we have all the possible chords. 
A rough idea of the proof in the non-extremal case is as follows: We find the $k$ th power of a path in $K_{1}$ by going around the cycle as many times as possible. Then we connect this path to $K_{2}$ with the use of a few extra vertices, then find the $k$ th power of a path in $K_{2}$, etc. However, for technical reasons, we will start by constructing the connecting paths between the subsequent cliques (for the last one $K_{s}$ the next one is $K_{1}$ ). This will be the first part of the proof in Sec. 5. In Sec. 6, we will take care of the exceptional vertices and make some adjustments to ensure that the distribution of the vertices inside each clique is perfect, i.e., there are the same number of vertices in each cluster of the clique. Finally, using Lemma 2.4, we string the vertices inside each clique into the $k$ th power of a path.

\section{Modifying the Clique Cover}

We remove $\frac{\sqrt{d}}{k+1} l$ (for simplicity we assume that this number is an integer) $(k+1)$ cliques from the clique cover. Let us denote the number of remaining $(k+1)$-cliques by $s$. Our goal in this section is to show that by slightly changing the remaining cliques and by redistributing the removed clusters, we can obtain a new clique cover in which $\frac{\sqrt{d}}{k+1} l(k+1)=\sqrt{d} l$ of the cliques are $(k+2)$-cliques and the remaining $s-\sqrt{d} l$ cliques are $(k+1)$-cliques.

Let us consider an arbitrary removed cluster $C$. If there is a $(k+1)$-clique $K$ in the current cover ( $C$ might not be the first cluster we redistribute) such that we have $\left(C, C^{\prime}\right) \in E\left(G_{r}\right)$ for every $C^{\prime} \in K$, then we just add $C$ to $K$, we have one more $(k+2)$ clique and we can move to the next removed cluster. Thus, we may assume that there is no $(k+1)$-clique $K$ with this property. Using this fact, (3.2), (3.3), and an easy calculation shows that the number of $(k+1)$-cliques $K$, for which

$$
\left|\left\{C^{\prime} \mid C^{\prime} \in K,\left(C, C^{\prime}\right) \in E\left(G_{r}\right)\right\}\right|=k,
$$

is at least $\left(1-d^{1 / 3}\right) s$. We consider only these $(k+1)$-cliques where the $(k+1)$ st cluster that is not a neighbor of $C$ is called a $C$-exchangable cluster. Indeed, these clusters are exchangable with $C$. Let us denote the set of $C$-exchangable clusters by $S$. Assume first that we have a $C^{\prime} \in S$ and $(k+1)$-cliques $K, K^{\prime}$ such that $C^{\prime} \in K$ and $\left(C^{\prime}, C^{\prime \prime}\right) \in E\left(G_{r}\right)$ for every $C^{\prime \prime} \in K^{\prime}$. Then again we are done since we remove $C^{\prime}$ from $K$ and add it to $K^{\prime}$, we add $C$ to $K$ and thus, we have one more $(k+2)$-clique. Hence, we may assume that there is no $C^{\prime}$ with this property.

However, in this case the fact that EC does not hold, (3.1), (3.2) and some computation imply that we can find cliques $K, K^{\prime}$ with $C_{1}=K \cap S, C_{2}=K^{\prime} \cap S$ such that

- $\left(C_{1}, C_{2}\right) \in E\left(G_{r}\right)$;

- there exists a cluster $C_{3} \in K \backslash C_{1}$ with $\left(C_{2}, C_{3}\right) \notin E\left(G_{r}\right)$;

- $N_{G_{r}}\left(C_{2}\right) \cap K=K \backslash C_{3}, N_{G_{r}}\left(C_{3}\right) \cap K^{\prime}=K^{\prime} \backslash C_{2}$.

Here, we also use the fact that $C_{3}$ is $C$-exchangable in two steps. Indeed, we remove $C_{2}$ from $K^{\prime}$ and add $C$ to it, we remove $C_{3}$ from $K$ and add $C_{2}$ to it, and now $C_{3}$ plays the role of $C$.

But then we exchange $C_{2}$ and $C_{3}$ among $K$ and $K^{\prime}$ and add $C$ to $K^{\prime}$, thus creating one more $(k+2)$-clique again. By repeating this procedure, we obtain a clique sequence 
$K_{1}, K_{2}, \ldots, K_{s}$ where the first $s^{\prime}=\sqrt{d} l$ cliques are $(k+2)$-cliques and the others are $(k+1)$-cliques.

\section{Connecting the Cliques}

To connect the cliques, first we will use only the $(k+1)$-clique $V_{1}^{i}, V_{2}^{i}, \ldots, V_{k+1}^{i}$ even if $1 \leq i \leq s^{\prime}$. For simplicity, we keep the notation $K_{1}, K_{2}, \ldots, K_{s}$ for these cliques.

We construct the connecting path between $K_{i}$ and $K_{i+1}$ for $1 \leq i \leq s$ (for $i=s$, $K_{i+1}=K_{1}$ ). Firstly, we determine the sequence of clusters from which the connecting path will use vertices. This sequence will be the square of a path in $G_{r}$ (however, it will not be a simple path).

We will repeatedly use the following fact, which is a consequence of (3.2).

Fact 5.1. Let $V_{1}, V_{2}, \ldots, V_{k}$ be $k$ arbitrary clusters in $G_{r}$. Then

$$
\left|N_{G_{r}}\left(V_{1}, V_{2}, \ldots, V_{k}\right)\right| \geq\left(\frac{1}{k+1}-3 k d\right) l \text {. }
$$

In other words, every set of $k$ clusters has a common neighborhood set of a size of roughly $\frac{l}{k+1}$.

Firstly, our goal is to define a sequence of $(k+1)$-cliques in $G_{r}$

$$
K^{0}, K^{1}, \ldots, K^{t}
$$

with the following properties:

- $K^{0}=K_{i}, K^{t}=K_{i+1}$,

- $\left|K^{j+1} \cap K^{j}\right|=k$ for every $0 \leq j \leq t-1$,

- $t=O\left(k^{2}\right)$.

For this purpose, if $K$ and $K^{\prime}$ are two $(k+1)$-cliques, for every cluster $C$ in $G_{r} \backslash\left(K \cup K^{\prime}\right)$ we determine a label $\ell_{K, K^{\prime}}(C)=(a, b), 0 \leq a \leq k+1,0 \leq b \leq k+1$ in the following way.

$$
a=\operatorname{deg}_{G_{r}}(C, K) \text { and } b=\operatorname{deg}_{G_{r}}\left(C, K^{\prime}\right) .
$$

We are going to construct the sequence in (5.1) in two steps. Firstly, we will construct two sequences of $(k+1)$-cliques

$$
A_{1}, A_{2}, \ldots, A_{t_{1}} \text { and } B_{1}, B_{2}, \ldots, B_{t_{2}}
$$

with the following properties:

(a) $A_{1}=K_{i}, B_{1}=K_{i+1}$;

(b) $\left|A_{j_{1}+1} \cap A_{j_{1}}\right|=k,\left|B_{j_{2}+1} \cap B_{j_{2}}\right|=k$ for every $0 \leq j_{1} \leq t_{1}-1,0 \leq j_{2} \leq t_{2}-1$;

(c) either

$$
\operatorname{deg}_{G_{r}}\left(C, A_{t_{1}}\right) \geq k \text { for every } C \in B_{t_{2}}
$$

or

$$
\operatorname{deg}_{G_{r}}\left(C^{\prime}, B_{t_{2}}\right) \geq k \text { for every } C^{\prime} \in A_{t_{1}}
$$


(d) $t_{1}+t_{2}=O\left(k^{2}\right)$.

Assume first that we have already constructed the two sequences in (5.2). Then in the second step of the construction of the clique sequence in (5.1), we construct a clique sequence

$$
D_{0}, D_{1}, \ldots, D_{k+1}
$$

which forms a gradual transition between $A_{t_{1}}$ and $B_{t_{2}}$. More precisely, first we assume that (5.3) holds in (c). We denote the clusters in $B_{t_{2}}$ by $V_{1}, V_{2}, \ldots, V_{k+1}$. Then we will have $D_{i} \subset A_{t_{1}} \cup B_{t_{2}}$ and $\left|D_{i} \cap B_{t_{2}}\right|=i$. The construction is the following. $D_{0}$ is just $A_{t_{1}}$. To get $D_{1}$, we add $V_{1}$ to $D_{0}$ and we remove the cluster from $D_{0}=A_{t_{1}}$ which is not adjacent to $V_{1}$ (if there is any). If all the clusters in $D_{0}$ are adjacent to $V_{1}$, then we remove an arbitrary cluster from $D_{0}$. In general, to obtain $D_{i+1}$ from $D_{i}, 0 \leq i \leq k$, we add $V_{i+1}$ to $D_{i}$ and remove the cluster from $D_{i} \cap D_{0}$ which is not adjacent to $V_{i+1}$ (if there is any). If all the clusters in $D_{i} \cap D_{0}$ are adjacent to $V_{i+1}$, then we remove an arbitrary cluster from $D_{i} \cap D_{0}$. If (5.4) holds in (c), then we reverse the same procedure; we construct the gradual transition backwards from $B_{t_{2}}$ (starting with $D_{k+1}$ ) to $A_{t_{1}}$ (ending at $D_{0}$ ).

The desired clique sequence in (5.1) is obtained in the following way.

$$
A_{1}, A_{2}, \ldots, A_{t_{1}}, D_{1}, D_{2}, \ldots, D_{k}, B_{t_{2}}, \ldots, B_{1} \text {. }
$$

For this sequence we use the notation in (5.1), so $t=t_{1}+t_{2}+k=O\left(k^{2}\right)$.

Thus, we only have to construct the two sequences in $(5,2)$ in the following way. $A_{1}=K_{i}, B_{1}=K_{i+1}$ and assume that $A_{1}, A_{2}, \ldots, A_{j_{1}}$ and $B_{1}, B_{2}, \ldots, B_{j_{2}}$ are already constructed, but (c) does not hold for $A_{j_{1}}$ and $B_{j_{2}}$. Our goal is to define a few more terms of the two sequences in such a way that we strictly increase the number of edges in $G_{r}$ between the two cliques, so that we get closer to (c). We repeat this procedure until (c) holds. It follows that in at most $O\left(k^{2}\right)$ steps, we can achieve (c), thus $t_{1}+t_{2}=O\left(k^{2}\right)$.

We may assume that there exists a $C_{1} \in A_{j_{1}}$ with $\operatorname{deg}\left(C_{1}, B_{j_{2}}\right) \leq k-1$ and a $C_{2} \in$ $B_{j_{2}}$ with $\operatorname{deg}\left(C_{2}, A_{j_{1}}\right) \leq k-1$, otherwise (c) holds. Denote $A=N_{G_{r}}\left(A_{j_{1}} \backslash C_{1}\right)$ and $B=N_{G_{r}}\left(B_{j_{2}} \backslash C_{2}\right)$. Fact 5.1 implies that $|A|,|B| \geq\left(\frac{1}{k+1}-3 k d\right) l$. For simplicity, we may assume that $|A|,|B| \leq \frac{l}{k+1}$ by removing some extra clusters.

Let us consider first the case where there is a $C \in G_{r} \backslash\left(A_{j_{1}} \cup B_{j_{2}}\right)$ with

$$
\ell_{A_{j_{1}}, B_{j_{2}}}(C)=(a, b) \text { with } a+b \geq 2 k+1 \text {. }
$$

In this case, either $a=k+1$ or $b=k+1$ (or both), say, $a=k+1$ (similar in the other case). To obtain $A_{j_{1}+1}$, we remove $C_{1}$ from $A_{j_{1}}$ and add $C$. We strictly increase the number of edges between the two cliques, thus we achieved our goal. Thus, we may assume that there is no cluster $C$ satisfying (5.5). However, in this case an easy computation using (3.2) shows that for most clusters $C \in G_{r} \backslash\left(A_{j_{1}} \cup B_{j_{2}}\right)$ we have

$$
\ell_{A_{j_{1}}, B_{j_{2}}}(C)=(a, b) \text { with } a+b=2 k .
$$

Indeed, the number of exceptional clusters for which (5.6) does not hold is $\leq 7(k+1) d l$. We delete these exceptional clusters from $A$ and $B$ and denote the resulting sets by $A^{\prime}$ and $B^{\prime}$. If we have a cluster $C \in A^{\prime}$ (similarly for $B^{\prime}$ ) with $\ell_{A_{j_{1}}, B_{j_{2}}}(C)=(k, k)$, then we 
obtain $A_{j_{1}+1}$ by removing $C_{1}$ from $A_{j_{1}}$ and adding $C$. Again we increased the number of edges between the two cliques.

Thus, we may assume that $\ell_{A_{j_{1}}, B_{j_{2}}}(C)=(k+1, k-1)$ for every $C \in A^{\prime}, \ell_{A_{j_{1}}, B_{j_{2}}}\left(C^{\prime}\right)=$ $(k-1, k+1)$ for every $C^{\prime} \in B^{\prime}$ and

$$
\frac{l}{k+1} \geq\left|A^{\prime}\right|,\left|B^{\prime}\right| \geq\left(\frac{1}{k+1}-10(k+1) d\right) l .
$$

This implies that we may also assume that $\operatorname{deg}\left(C_{1}, B_{j_{2}}\right)=k-1$ and $\operatorname{deg}\left(C_{2}, A_{j_{1}}\right)=$ $k-1$.

Next, we show that we may assume that $\left.G_{r}\right|_{A^{\prime}}$ and $\left.G_{r}\right|_{B^{\prime}}$ are almost complete graphs with an almost empty bipartite graph between them. In fact, firstly, for every $C \in A^{\prime}$, we have $\operatorname{deg}_{\bar{G}_{r}}\left(C, A^{\prime}\right) \leq 7(k+1) d l$ (and similarly for $B^{\prime}$ ). Otherwise, we obtain $A_{j_{1}+1}$ by removing $C_{1}$ and adding $C$, and we would have more than $7(k+1) d l$ clusters $C^{\prime}$ with $\ell_{A_{j_{1}}, B_{j_{2}}}\left(C^{\prime}\right)=(k, k-1)$ which is impossible. Furthermore, in case

$$
d_{G_{r}}\left(A^{\prime}, B^{\prime}\right) \geq \sqrt{d}
$$

it is not hard to see that there exist clusters

$$
\left\{C^{1}, C^{2}, \ldots, C^{k+1}\right\} \subset A^{\prime} \text { and }\left\{C^{k+2}, C^{k+3}, \ldots, C^{2(k+1)}\right\} \subset B^{\prime}
$$

such that these $2(k+1)$ clusters induce a $2(k+1)$-clique in $G_{r}$. Then similarly as above we form a gradual transition from $A_{j_{1}}$ to the $(k+1)$-clique $\left\{C^{1}, C^{2}, \ldots, C^{k+1}\right\}$ and this is $A_{t_{1}}$. This is similar for $B_{t_{2}}$ and clearly (c) is satisfied.

Next, we show that we may assume that for most of the clusters in $B^{\prime}$, the $k-1$ clusters in $A_{j_{1}}$ are precisely the same. For this purpose we show first that we may assume that for most clusters in $B^{\prime}$, the $k-1$ neighbors are in $A_{j_{1}} \backslash C_{1}$. Indeed, we take a cluster $C$ in $A^{\prime}$ with $\operatorname{deg}_{G_{r}}\left(C, B^{\prime}\right) \leq \sqrt{d}\left|B^{\prime}\right|$ (the fact that (5.7) does not hold implies that $C$ exists). Then we define $A_{j_{1}+1}=\left(A_{j_{1}} \backslash C_{1}\right) \cup C$, and indeed for at least $(1-\sqrt{d})\left|B^{\prime}\right|$ clusters in $B^{\prime}$, the $k-1$ neighbors are in $A_{j_{1}+1} \backslash C\left(C\right.$ plays the role of $C_{1}$ now). For simplicity of notation, let us assume that this is already true for $A_{j_{1}}$ and $B^{\prime}$. So for at least $(1-\sqrt{d})\left|B^{\prime}\right|$ clusters $C \in B^{\prime}, N_{G_{r}}(C) \cap A_{j_{1}}$ is a set of $k-1$ clusters in $A_{j_{1}} \backslash C_{1}$. Among the possible $\left(\begin{array}{c}k \\ k-1\end{array}\right)$ sets, we consider the one which occurs the most often as $N_{G_{r}}(C) \cap A_{j_{1}}$ for these clusters $C \in B^{\prime}$. We show that this set (denoted by $E$ ) occurs as $N_{G_{r}}(C) \cap A_{j_{1}}$ for at least $\left(1-d^{1 / 3}\right)\left|B^{\prime}\right|$ clusters $C \in B^{\prime}$. Assume indirectly that this is not the case. Denote the cluster in $A_{j_{1}} \backslash\left(E \cup C_{1}\right)$ by $C_{1}^{\prime}$, so $A_{j_{1}}=E \cup C_{1} \cup C_{1}^{\prime}$. We consider $N_{G_{r}}(E)$. We know that

$$
\left|N_{G_{r}}(E)\right| \geq\left(\frac{2}{k+1}-3 k d\right) l, A^{\prime} \subset N_{G_{r}}(E)
$$

and

$$
\left|N_{G_{r}}(E) \cap B^{\prime}\right|<\left(1-d^{1 / 3}\right)\left|B^{\prime}\right| \leq\left(1-d^{1 / 3}\right) \frac{l}{k+1} .
$$

We find clusters $X_{1}, X_{2}, Y$ with the following properties:

(1) $X_{1} \in N_{G_{r}}(E) \backslash\left(A^{\prime} \cup B^{\prime}\right), X_{2} \in A^{\prime}, Y \in B^{\prime} \cap N_{G_{r}}(E)$; 
(2) $\left(X_{1}, X_{2}\right),\left(X_{1}, Y\right),\left(X_{1}, C_{1}\right),\left(X_{1}, C_{1}^{\prime}\right) \in E\left(G_{r}\right)$;

(3) $\operatorname{deg}\left(X_{1}, B_{j 2}\right) \geq k$.

$(3.1),(3.2),(5.8)$ and the fact that (5.7) does not hold imply that we can easily achieve (1) and (2). If we could not achieve (3), then we would have a set $A^{\prime \prime}$ of clusters such that $A^{\prime} \subset A^{\prime \prime} \subset N_{G_{r}}(E) \backslash B^{\prime}$, for every cluster $C \in A^{\prime \prime}$, we have $\ell_{A_{j_{1}}, B_{j_{2}}}(C)=(k+1, k-1)$ and $\left|A^{\prime \prime}\right| \geq\left(1+\frac{d^{1 / 3}}{2}\right) \frac{l}{k+1}$. But this implies that $d_{G_{r}}\left(A^{\prime \prime}, B^{\prime}\right) \geq \sqrt{d}$, and similar to the case when (5.7) holds, we get the desired clique sequence.

Thus, we may assume that we can pick clusters $X_{1}, X_{2}, Y$ satisfying (1), (2), and (3). In this case

$$
A_{j_{1}+1}=\left(A_{j_{1}} \backslash C_{1}\right) \cup X_{2}, A_{j_{1}+2}=\left(A_{j_{1}+1} \backslash C_{1}^{\prime}\right) \cup X_{1} \text { and } A_{j_{1}+3}=\left(A_{j_{1}+2} \backslash X_{2}\right) \cup Y
$$

It is easy to check that this construction is good and that we strictly increase the number of edges between the two cliques.

We define $B^{\prime \prime}=B^{\prime} \cap N_{G_{r}}(E)$, so by the above, we have $\left|B^{\prime \prime}\right| \geq\left(1-d^{1 / 3}\right)\left|B^{\prime}\right|$. In $E$ we consider the cluster $C_{1}^{\prime \prime}$ for which $\operatorname{deg}\left(C_{1}^{\prime \prime}, B_{j_{2}}\right)$ is the smallest. Put $E^{\prime}=E \backslash C_{1}^{\prime \prime}$. We consider $N_{G_{r}}\left(E^{\prime}\right)$. We have $\left|N_{G_{r}}\left(E^{\prime}\right)\right| \geq\left(\frac{3}{k+1}-3 k d\right) l$ (using $k \geq 3$ ), so

$$
\left|N_{G_{r}}\left(E^{\prime}\right) \backslash\left(A^{\prime} \cup B^{\prime \prime}\right)\right| \geq\left(\frac{1}{k+1}-3 k d\right) l .
$$

We find clusters $X_{1}, X_{2}, Y_{1}, Y_{2}, Y_{3}$ with the following properties:

- $X_{1}, X_{2} \in N_{G_{r}}\left(E^{\prime}\right) \backslash\left(A^{\prime} \cup B^{\prime \prime}\right), Y_{1}, Y_{2}, Y_{3} \in B^{\prime \prime}$;

- $X_{1}, X_{2}, Y_{1}, Y_{2}, Y_{3}$ form a 5-clique in $G_{r}$;

- $X_{1}, X_{2}, C_{1}, C_{1}^{\prime}$ form a 4-clique in $G_{r}$.

Using (3.1), (3.2), (5.9) and the fact that EC does not hold, we can indeed choose these clusters with these properties. Then

$$
\begin{gathered}
A_{j_{1}+1}=\left(A_{j_{1}} \backslash C_{1}^{\prime \prime}\right) \cup X_{2}, A_{j_{1}+2}=\left(A_{j_{1}+1} \backslash C_{1}^{\prime}\right) \cup X_{1}, A_{j_{1}+3}=\left(A_{j_{1}+2} \backslash C_{1}\right) \cup Y_{1}, \\
A_{j_{1}+4}=\left(A_{j_{1}+3} \backslash X_{1}\right) \cup Y_{2}, \text { and } A_{j_{1}+5}=\left(A_{j_{1}+4} \backslash X_{2}\right) \cup Y_{3} .
\end{gathered}
$$

Again, the construction is good and we strictly increase the number of edges between the two cliques.

Thus, we may assume that we have the desired clique sequence in (5.1). Given this clique sequence, we obtain the sequence of clusters from which the connecting path will use vertices in the following way. We start by going around $K^{0}=K_{i}$, so by $V_{1}^{i}, V_{2}^{i}, \ldots, V_{k+1}^{i}$. We start a second cycle and stop at the last cluster before the cluster in $K^{0} \backslash K^{1}$. The next cluster is the cluster in $K^{1} \backslash K^{0}$, then we go around $K^{1}$ once and in the second cycle we stop at the last cluster before the cluster in $K^{1} \backslash K^{2}$. The next cluster is the cluster in $K^{2} \backslash K^{1}$, etc. We continue in this fashion to obtain a sequence of clusters (note that this sequence contains repetitions)

$$
C_{1}, C_{2}, \ldots, C_{t^{\prime}}
$$


where $C_{j}=V_{j}^{i}$ for $1 \leq j \leq k+1$; the last $k+1$ clusters are the clusters $V_{1}^{i+1}, V_{2}^{i+1}, \ldots$, $V_{k+1}^{i+1}$ in some permutation and $t^{\prime}=O\left(k^{2}\right)$.

However, for technical reasons we would like to end the sequence with $V_{1}^{i+1}$, $V_{2}^{i+1}, \ldots, V_{k+1}^{i+1}$ in this order. For this purpose it is sufficient to show that if $V_{1}, V_{2}, \ldots, V_{k+1}$ is an arbitrary permutation of $V_{1}^{i+1}, V_{2}^{i+1}, \ldots, V_{k+1}^{i+1}$, then we can change the order to

$$
V_{1}, \ldots, V_{j-1}, V_{j^{\prime}}, V_{j+1}, \ldots, V_{j^{\prime}-1}, V_{j}, V_{j^{\prime}+1}, \ldots, V_{k+1}
$$

for any $1 \leq j<j^{\prime} \leq k+1$. We separate two cases depending on whether $K_{i+1}$ is a $(k+2)$-clique or a $(k+1)$-clique.

Case $1.1 \leq i+1 \leq s^{\prime}$, so $K_{i+1}$ is a $(k+2)$-clique. In this case we will use $V_{k+2}^{i+1}$. The sequence of clusters is as follows:

$$
\begin{gathered}
V_{1}, \ldots, V_{k+1}, V_{1}, \ldots, V_{j-1}, V_{k+2}^{i+1}, V_{j+1}, \ldots, V_{k+1}, V_{1}, \ldots, V_{j-1}, V_{k+2}^{i+1}, V_{j+1}, \ldots, V_{j^{\prime}-1}, \\
V_{j}, V_{j^{\prime}+1}, \ldots, V_{k+1}, V_{1}, \ldots, V_{j-1}, V_{k+2}^{i+1}, V_{j+1}, \ldots, V_{j^{\prime}-1}, V_{j}, V_{j^{\prime}+1}, \ldots, V_{k+1} \\
V_{1}, \ldots, V_{j-1}, V_{j^{\prime}}, V_{j+1}, \ldots, V_{j^{\prime}-1}, V_{j}, V_{j^{\prime}+1}, \ldots, V_{k+1}
\end{gathered}
$$

as desired.

Thus, we may assume in this case that we have a sequence of clusters

$$
C_{1}, C_{2}, \ldots, C_{t^{\prime \prime}}
$$

which form the square of a path in $G_{r}$ and where $C_{j}=V_{j}^{i}$ for $1 \leq j \leq k+1$ and $C_{t^{\prime \prime}-j}=$ $V_{(k+1)-j}^{i+1}$ for $0 \leq j \leq k$ with $t^{\prime \prime}=O\left(k^{2}\right)$. We also define

$$
C_{0}=V_{k+2}^{i}, C_{-1}=V_{k+1}^{i}, C_{-2}=V_{k}^{i}, \ldots, C_{-k+1}=V_{3}^{i}
$$

(or $C_{0}=V_{k+1}^{s}, C_{-1}=V_{k}^{s}, C_{-2}=V_{k-1}^{s}, \ldots, C_{-k+1}=V_{2}^{s}$ if $K_{i+1}=K_{1}$ so $i=s$ )

and similarly,

$$
C_{t^{\prime \prime}+1}=V_{k+2}^{i+1}, C_{t^{\prime \prime}+2}=V_{1}^{i+1}, \ldots, C_{t^{\prime \prime}+k}=V_{k-1}^{i+1} .
$$

Now we choose a vertex $p_{j}$ from each cluster $C_{j}, 1 \leq j \leq t^{\prime \prime}$, such that $p_{j}$ is connected to all $p_{j^{t}}$ with $1 \leq\left|j^{\prime}-j\right| \leq k$. They will also have the following additional properties for all $j, 1 \leq j \leq k$ :

$$
\begin{gathered}
\left|N\left(p_{1}, p_{2}, \ldots, p_{j}\right) \cap C_{j-k}\right|>(d-\varepsilon)^{j} L, \\
\left|N\left(p_{t^{\prime \prime}}, p_{t^{\prime \prime}-1}, \ldots, p_{t^{\prime \prime}+1-j}\right) \cap C_{t^{\prime \prime}+1+k-j}\right|>(d-\varepsilon)^{j} L,
\end{gathered}
$$

which ensure that they can later be extended to the $k$ th power of a Hamiltonian cycle of $G^{\prime}$.

We will select them one-by-one with a greedy procedure. We maintain that $t^{\prime \prime}+2 k$ sets $H_{j, j^{\prime}}$ from which the points will be selected. We start with $H_{0, j^{\prime}}=C_{j^{\prime}}, 1-k \leq j^{\prime} \leq$ $t^{\prime \prime}+k$. 
Then, when selecting the point $p_{j}$ from $H_{j-1, j}, 1 \leq j \leq t^{\prime \prime}$, we choose one with the following property:

$$
\operatorname{deg}\left(p_{j}, H_{j-1, j^{\prime}}\right)>(d-\varepsilon)\left|H_{j-1, j^{\prime}}\right| \text { for all } j^{\prime} \neq j,\left|j^{\prime}-j\right| \leq k .
$$

This holds for all but at most $2 k \varepsilon\left|C_{j}\right|$ vertices in $H_{j-1, j}$, so we can choose such a $p_{j} \in H_{j-1, j}$. (Here, we used $(d-\varepsilon)^{k}>\varepsilon$. )

Then we update the sets $H$ as follows.

$$
H_{j, j^{\prime}}= \begin{cases}H_{j-1, j^{\prime}} \cap N\left(p_{j}\right), & \text { if } 1 \leq\left|j^{\prime}-j\right| \leq k, \\ H_{j-1, j^{\prime}} \backslash\left\{p_{j}\right\}, & \text { otherwise. }\end{cases}
$$

Note that we did not choose any points from the sets $H_{t^{\prime \prime}, j^{\prime}}$ for $j^{\prime}<1$ and $j^{\prime}>t^{\prime \prime}$; this selection will be done later. We will refer later to this point selection procedure described above as the standard greedy procedure.

Case 2. $s^{\prime}<i+1 \leq s$, so $K_{i+1}$ is a $(k+1)$-clique. The main idea is the same but in (5.10), we have to replace $V_{k+2}^{i+1}$ with something else. Fact 5.1 and the fact that EC does not hold guarantee that in $G$ there exists a complete bipartite graph, say, between sets $U_{j}$ and $W_{j}$ such that $\left|U_{j}\right|=\left|W_{j}\right|=f(k)$ (where $f(k)$ is sufficiently large compared to $k$ ). Also, for all the $k$ clusters $C \in\left\{V_{1}, V_{2}, \ldots, V_{j-1}, V_{j+1}, \ldots, V_{k+1}\right\}$ we have

$$
\left|N_{G}\left(U_{j} \cup W_{j}\right)\right| \geq \sqrt{\varepsilon}|C| \text {. }
$$

Then we add $U_{j}$ to $V_{j}$ and $W_{j}$ will play the role of $V_{k+2}^{i+1}$ in (5.10) (although it is not a cluster). Hence, in (5.11), some of the $C_{j}$-s are not clusters but they come from these complete bipartite graphs. In this case, we define

$$
C_{0}=V_{k+1}^{i}, C_{-1}=V_{k}^{i}, C_{-2}=V_{k-1}^{i}, \ldots, C_{-k+1}=V_{2}^{i}
$$

or

$$
C_{0}=V_{k+2}^{s^{\prime}}, C_{-1}=V_{k+1}^{s^{\prime}}, C_{-2}=V_{k}^{s^{\prime}}, \ldots, C_{-k+1}=V_{3}^{s^{\prime}} \text { if } i=s^{\prime}
$$

and similarly,

$$
C_{t^{\prime \prime}+1}=V_{1}^{i+1}, C_{t^{\prime \prime}+2}=V_{2}^{i+1}, \ldots, C_{t^{\prime \prime}+k}=V_{k}^{i+1} .
$$

It is not hard to see that with minor modifications the greedy procedure in Case 1 goes through in this case as well.

\section{Adjustments and the Handling of the Exceptional Vertices}

We already have an exceptional set $V_{0}$ of vertices in $G$. We add some more vertices to $V_{0}$ to achieve super-regularity. From a cluster $V_{j}^{i}$ in a clique $K_{i}$, we remove all vertices $v$ for which there exists an $j^{\prime}$ with $1 \leq j^{\prime} \leq k+1\left(k+2\right.$ if $\left.1 \leq i \leq s^{\prime}\right), j^{\prime} \neq j$ such that

$$
\operatorname{deg}\left(v, V_{j^{\prime}}^{i}\right) \leq(d-\varepsilon)\left|V_{j^{\prime}}^{i}\right|
$$

$\varepsilon$-regularity guarantees that at most $(k+1) \varepsilon\left|V_{j}^{i}\right| \leq(k+1) \varepsilon L$ of such vertices exist in each cluster $V_{j}^{i}$. 
We may have a small discrepancy in the number of remaining vertices in each clique $K_{i}$ (we removed some form the connecting paths and some from the last step). By removing extra vertices from certain clusters (and put them into the exceptional set $V_{0}$ ), we achieve that each cluster has exactly $L^{\prime}$ vertices. (We will still use the notation $V_{0}$ for the enlarged exceptional set.) We still have $\left|V_{0}\right| \leq 6 k(k+1) d n$.

Next, we take care of the vertices in $V_{0}$. For each vertex $v \in V_{0}$, we find all $K_{i}$ 's such that if $i>s^{\prime}$, then

$$
\operatorname{deg}(v, C) \geq d|C| \text { for all } C \in K_{i}
$$

and if $1 \leq i \leq s^{\prime}$, then there exist $(k+1)$ clusters $C \in K_{i}$ such that

$$
\operatorname{deg}(v, C) \geq d|C|
$$

Inequality (1.1) easily shows that we have at least $d^{3 / 4} s$ such cliques for each $v \in V_{0}$. We assign each $v \in V_{0}$ to one of these cliques in such a way that we do not assign too many vertices to a particular clique. It is easy to see that an assignment is possible in which no clique is assigned more than $d^{1 / 3} L^{\prime}$ vertices.

Now let us take the first vertex $v \in V_{0}$ and let us assume first that it is assigned to $K_{i}$ with $i>s^{\prime}$. We will add $v$ to the connecting path between $K_{i-1}$ and $K_{i}$ by also using some vertices from $K_{i}$ in such a way that the extended path is still extendable to the $k$ th power of a Hamiltonian cycle, and we use the same number of vertices from each cluster in $K_{i}$ (in fact exactly three from each cluster).

Let us denote the connecting path between $K_{i-1}$ and $K_{i}$ by $p_{1}, p_{2}, \ldots, p_{k^{\prime}}$. We extend this path in essentially the same way as in the previous section by using the standard greedy procedure with vertices $p_{k^{\prime}+1}, p_{k^{\prime}+2}, \ldots, p_{k^{\prime \prime}}$, where $k^{\prime \prime}=k^{\prime}+3(k+1)+1$. We go around the clusters of the clique three times. The only change in the procedure described in the previous section is that the new points $p_{j}, k^{\prime}+1 \leq j \leq k^{\prime \prime}$ should have the additional property

$$
\left|V_{\ell} \cap N(v) \bigcap\left\{N\left(p_{j}\right): k^{\prime}+1 \leq j \leq k^{\prime \prime}, p_{j} \notin V_{\ell}\right\}\right|>d^{3(k+1)+1} L^{\prime} \text { for each } V_{\ell} \in K_{i}
$$

This guarantees that the new vertex $v$ can be added as $p_{k^{\prime}+2(k+1)+1}$, and the previous and next $k$ vertices can be chosen from $N(v)$.

In case $v$ is assigned to $K_{i}$ with $1 \leq i \leq s^{\prime}$, we perform the following. We denote the clique of the $(k+1)$ clusters $C$ for which $(6.1)$ holds for $v$ by $K_{i}^{\prime}$ and put $C^{\prime}=K_{i} \backslash K_{i}^{\prime}$. We can extend the connecting path between $K_{i-1}$ and $K_{i}$ so that now it ends with the clusters in $K_{i}^{\prime}$. Just as above we go around $K_{i}^{\prime} 3$ times such that we can include $v$ on the path. The only problem is that we created a small discrepancy among the number of remaining vertices in the clusters in $K_{i}$. To avoid this complication we use the cluster $C^{t}$ as well. Indeed, we extend the path so that now it ends with $V_{k+1}^{i}$ and we go around $K_{i}$ a few times, skipping the cluster with the smallest number of remaining vertices until we have the same number of remaining vertices in each cluster.

Finally, because $\left|V_{0}\right|$ is quite large, we cannot just repeat this procedure for all vertices in $V_{0}$, since we might hurt the super-regularity. Note that we never hurt the $\varepsilon$ regularity. Therefore, we perform the following. We define $\kappa$ as $\varepsilon \ll \kappa \ll d$. We maintain another set $Q$ beside $V_{0}$. Initially $Q=\emptyset$. After handling $\lfloor\kappa n\rfloor$ vertices from $V_{0}$, we update $Q$ in the following way. From a cluster $V_{j}^{i}$ in a clique $K_{i}$, we remove 
all vertices $v$ and add them to $Q$ for which there exists a $j^{\prime}$ with $1 \leq j^{\prime} \leq k+1(k+2$ if $\left.1 \leq i \leq s^{\prime}\right), j^{\prime} \neq j$ such that $\operatorname{deg}\left(\nu, V_{j^{\prime}}^{i}\right) \leq(d-\varepsilon)\left|V_{j^{\prime}}^{i}\right|$. Here, we only consider the remaining vertices in a cluster. We also remove some extra vertices to make sure that we have the same number of vertices remaining in the clusters in $K_{i}$. $\varepsilon$-regularity guarantees that we added at most $(k+1) \varepsilon n$ vertices to $Q$. Then we handle the vertices in $Q$ exactly the same way as the exceptional vertices above. Next we handle the next $[\kappa n]$ vertices of $V_{0}$. After this we update $Q$ and we handle the new vertices in $Q$, etc.

Thus, we are left with the following situation: In each clique $K_{i}$ we have the same number of remaining vertices in each cluster (including $V_{k+2}^{i}$ if $1 \leq i \leq s^{\prime}$ ). On the connecting path between $K_{i-1}$ and $K_{i}$, the last $k$ vertices have many common neighbors in $V_{1}^{i}$ (in $V_{k+2}^{i}$ if $1 \leq i \leq s^{\prime}$ ), the last $k-1$ vertices have many common neighbors in $V_{2}^{i}$ (in $V_{1}^{i}$ if $1 \leq i \leq s^{\prime}$ ), etc., and finally the last vertex has many neighbors in $V_{k}^{i}$ (in $V_{k-1}^{i}$ if $\left.1 \leq i \leq s^{\prime}\right)$. On the connecting path between $K_{i}$ and $K_{i+1}$, the first $k$ vertices have many common neighbors in $V_{k+1}^{i}$ (in $V_{k+2}^{i}$ if $1 \leq i \leq s^{\prime}$ ), the first $k-1$ vertices many common neighbors in $V_{k}^{i}$ (in $V_{k+1}^{i}$ if $1 \leq i \leq s^{\prime}$ ), etc., and finally the first vertex has many neighbors in $V_{2}^{i}$ (in $V_{3}^{i}$ if $1 \leq i \leq s^{\prime}$ ). These properties guarantee that by using Lemma 2.4 we can close the $k$ th power of a Hamiltonian cycle inside each clique.

\section{The Extremal Case}

In this section we assume that the extremal case $(\mathrm{EC})$ is satisfied so we have an $A \subset$ $V(G)$ with $|A|=\left\lfloor\frac{n}{k+1}\right\rfloor$ and $d(A)<\alpha$. Let us assume first that we have the following special case: There exists a partition

$$
V(G)=A_{1} \cup A_{2} \cup \ldots \cup A_{k+1}
$$

with $\left|A_{i}\right|=\left\lfloor\frac{n}{k+1}\right\rfloor$ for $1 \leq i \leq k$ and $d\left(A_{i}\right)<\alpha$ for $1 \leq i \leq k+1$. In each $A_{i}$, we can have at most $\alpha^{2 / 3}\left|A_{i}\right|$ exceptional vertices $v \in A_{i}$ for which we have

$$
\operatorname{deg}\left(v, A_{i}\right) \geq \alpha^{1 / 3}\left|A_{i}\right|
$$

We call these exceptional vertices in $A_{i} i$-bad. For simplicity, let us assume first that we have no $i$-bad vertices for any $1 \leq i \leq k+1$. In this case the only problem is that $A_{k+1}$ could be slightly larger than the other $A_{i}$-s, otherwise Lemma 2.4 would find the $k$ th power of a Hamiltonian cycle. For this purpose, using Lemma 2.5 , we can find a matching of size $\left|A_{k+1}\right|-\left\lfloor\frac{n}{k+1}\right\rfloor$ in $A_{k+1}$. Indeed, from (1.1), we have

$$
\delta\left(\left.G\right|_{A_{k+1}}\right) \geq\left|A_{k+1}\right|-\left\lfloor\frac{n}{k+1}\right\rfloor .
$$

We contract these edges into vertices, where the neighbors of a new vertex are the common neighbors of the two endpoints of the corresponding edge. Since every $\left(A_{i}, A_{j}\right)$ pair is $(\varepsilon, \delta)$ super-regular with the appropriate choice of parameters (say, $\varepsilon=\alpha^{1 / 6}, \delta=$ $1-\alpha^{1 / 3}$ ) and we have the same number of vertices in each $A_{i}$, by using the Blow-up Lemma (Lemma 2.4), we can find the $k$ th power of a Hamiltonian cycle.

When we have bad vertices satisfying (7.1), the main idea is the same but we have to handle the bad vertices first. More precisely, we have to eliminate a special type of 
bad vertices. For a vertex $v \in A_{i}$, we say that it is $j$-exceptional $(j \neq i)$, if

$$
\operatorname{deg}\left(v, A_{j}\right) \leq \frac{\alpha^{1 / 3}}{2}\left|A_{j}\right| .
$$

Note that if a vertex $v \in A_{i}$ is $j$-exceptional for some $j \neq i$, then it is $i$-bad. Firstly, we have to eliminate the $i$-exceptional vertices for every $1 \leq i \leq k+1$. The other bad vertices do not cause any further complications.

We may assume that for every $1 \leq i \leq k+1$, there are either no $i$-bad vertices or no $i$-exceptional vertices in the other $A_{j}$-s $(j \neq i)$. Otherwise, we could exchange an $i$-bad vertex in $A_{i}$ with an $i$-exceptional vertex in $A_{j}$. In this way we decrease the number of $i$-bad vertices. By iterating this procedure, there are either no more $i$-bad vertices or no more $i$-exceptional vertices left.

If $\left|A_{k+1}\right|>\left\lfloor\frac{n}{k+1}\right\rfloor$, then similarly as above, we have to find a matching to adjust the differences in the sizes of the sets $A_{i}$. However, if $A_{k+1}$ contains a $j$-exceptional vertex for some $j \neq k+1$, then we have to be careful since one of the endpoints of an edge could be a $j$-exceptional vertex. Then the endpoints do not have a large common neighborhood set in $A_{j}$. For this purpose we perform the following. We remove a $j$ exceptional vertex from $A_{k+1}$ and we add it to $A_{j}$ (we still keep the notation $A_{1}, A_{2}, \ldots$, $\left.A_{k+1}\right)$. We iterate this procedure; if there exists an $A_{i}$ with $\left|A_{i}\right|>\left\lfloor\frac{n}{k+1}\right\rfloor$ and a $j$ exceptional vertex for some $j \neq i$, then we remove the vertex from $A_{i}$ and we add it to $A_{j}$. Since we always decrease the number of $j$-exceptional vertices, eventually this process has to stop. Thus, we may assume that if $\left|A_{i}\right|>\left\lfloor\frac{n}{k+1}\right\rfloor$ holds for $A_{i}$, then $A_{i}$ does not contain $j$-exceptional vertices for $j \neq i$. For each such $A_{i}$, we choose a matching $M_{i}$ of size $\left|A_{i}\right|-\left\lfloor\frac{n}{k+1}\right\rfloor$ in $A_{i}$ as above. Indeed, we can always choose the edges in the matching $M_{i}$ in such a way that, for an edge in the match, the two endpoints are either both good (not $i$-bad) or one of them is good and the other is $i$-bad but with a common neighborhood of size at least $\frac{\alpha^{1 / 3}}{4}\left|A_{j}\right|$ in every $A_{j}$ for $j \neq i$. Before we contract these edges into vertices and finish with the Blow-up Lemma as above, we first have to eliminate the $i$-exceptional vertices for every $1 \leq i \leq k+1$.

Consider an $1 \leq i \leq k+1$. By the above remark if there exist $i$-exceptional vertices in other $A_{j}$ 's (say, we have $x_{i}$ of them), then we do not have $i$-bad vertices. If possible we take a set $P_{i}$ of $x_{i}$ paths of length 2 which are vertex-disjoint from each other and from all the matchings $M_{i}$, where the 2 endpoints are in $A_{i}$ and the middle point is either in $A_{i}$ or it is $i$-exceptional in some $A_{j}, j \neq i$. Using (1.1), Lemma 2.5 and the fact that there are no $i$-bad vertices, it is not hard to see that the only case when it is not possible to find these $x_{i}$ paths of length 2 is when $x_{i}=1$ (say, this exceptional vertex in $A_{j}$ is denoted by $v_{i}$ ).

Let us assume first that $x_{i}>1$ for every $1 \leq i \leq k+1$ so that we can find these sets of paths $P_{i}$. Taking the natural ordering $A_{1}, A_{2}, \ldots, A_{k+1}$, we can start building the $k$ th power of the Hamiltonian cycle by the standard greedy strategy (see Sec. 5) going around the cycle. Consider a $1 \leq i \leq k+1$. If the middle point of one such path in $P_{i}$ is an $i$-exceptional vertex in some $A_{j}$, after a few cycles we can easily put this path (or more precisely the three vertices on the path) with the greedy strategy on the $k$ th power of the Hamiltonian cycle being built. Furthermore, the part of the $k$ th power of the Hamiltonian cycle that we construct is always vertex-disjoint from all the matchings $M_{i}$ and all the other remaining paths in $\cup_{i} P_{i}$. If the middle point is in $A_{i}$, then 
we exchange this middle point with an arbitrary $i$-exceptional vertex in some $A_{j}, j \neq i$ (different ones for different paths). Again, we put this path of length 2 on the $k$ th power of the Hamiltonian cycle being built. Iterating this for all $1 \leq i \leq k+1$, we eliminate all $i$-exceptional vertices. Then we contract the edges into vertices in the matchings $M_{i}$ and we finish with the Blow-up Lemma.

For each $i$ with $x_{i}=1$, we choose a path $p_{i}$ of length 2 which is vertex-disjoint from all the matchings $M_{i}$ and all other paths and where $v_{i}$ is the middle vertex, one endpoint $u_{i}$ is in $A_{i}$ and the other endpoint $\bar{u}_{i}$ is good in $A_{j}$. We can put this path $p_{i}$ on the $k$ th power of the Hamiltonian cycle being built, but unfortunately, this will change the natural $A_{1}, A_{2}, \ldots, A_{k+1}$ ordering. In order to change the ordering back to the original, we will need an edge $\left(w_{i}, \overline{w_{i}}\right)$ inside $A_{i}$ that is vertex-disjoint from all the matching edges and all other paths. We shall perform the following. Assuming $i<j$, first we put $u_{i}$ and $v_{i}$ on the $k$ th power of the Hamiltonian cycle in the natural $A_{1}, A_{2}, \ldots, A_{k+1}$ ordering. However, in the next cycle we jump over $A_{i}, A_{i+1}, \ldots, A_{j-1}$; from $A_{j}$ we pick $\overline{u_{i}}$ (and we use the $\left(v_{i}, \bar{u}_{i}\right)$ edge for the necessary connection), then we have $A_{j-1}, A_{j-2}, \ldots, A_{i}$. Then we pick $A_{j+1}$ and we continue in the natural ordering. More precisely, in the second cycle we pick one vertex from each set in the following sequence ( $\overline{u_{i}}$ from $\left.A_{j}\right)$ :

$$
A_{1}, \ldots, A_{i-1}, A_{j}, A_{j-1}, \ldots, A_{i+1}, A_{i}, A_{j+1}, \ldots, A_{k+1} .
$$

After a few cycles in this ordering, we can change back the ordering to the original using the edge $\left(w_{i}, \overline{w_{i}}\right)$.

We repeat this procedure for all $1 \leq i \leq k+1$ with $x_{i}=1$. Then we eliminate the exceptional vertices for all $1 \leq i \leq k+1$ with $x_{i}>1$ with the above procedure. Then we contract the edges into vertices in the matchings $M_{i}$ and we finish with the Blow-up Lemma.

In the general extremal case, we first have an $A_{1} \subset V(G)$ with $\left|A_{1}\right|=\left\lfloor\frac{n}{k+1}\right\rfloor$ and $d\left(A_{1}\right)<\alpha$. If possible, we take an $A_{2} \subset V(G) \backslash A_{1}$ in the leftover with $\left|A_{2}\right|=\left\lfloor\frac{n}{k+1}\right\rfloor$ and $d\left(A_{2}\right)<\alpha$. We may continue this process unless there is no $A_{l+1} \subset V(G) \backslash\left(A_{1} \cup \ldots \cup A_{l}\right)$ with $\left|A_{l+1}\right|=\left\lfloor\frac{n}{k+1}\right\rfloor$ and $d\left(A_{l+1}\right)<\alpha$. Put $B=V(G) \backslash\left(A_{1} \cup \ldots \cup A_{l}\right)$. If $l=k$, we get back the special extremal case just discussed (with somewhat worse $\alpha$ ). Assume first that $l \leq k-2$. We define $i$-bad vertices in $A_{i}, 1 \leq i \leq l$ just as in (7.1). In $B$, the bad vertices are vertices $v$ with

$$
\operatorname{deg}\left(v, A_{1} \cup \ldots \cup A_{l}\right) \leq\left(1-\alpha^{1 / 3}\right)\left|A_{1} \cup \ldots \cup A_{l}\right| .
$$

Again let us assume first that there are no bad vertices and that $|B|=(k-l+1)\left\lfloor\frac{n}{k+1}\right\rfloor$. Since there is no $A \subset B$ with $|A|=\left\lfloor\frac{n}{k+1}\right\rfloor=\left\lfloor\frac{1}{k-1+1}|B|\right\rfloor$ and $d(A)<\alpha,\left.G\right|_{B}$ does not satisfy the extremal condition for $k-l$. Therefore, the method described in the previous sections succeeds in finding the $(k-l)$ th power of a Hamiltonian cycle $H$ in $B$. Actually, the method in this paper only works for $k-l \geq 3$, but the same result is proved in [13] with $k-l=2$. Denote $H=p_{1}, p_{2}, \ldots, p_{|B|}$.

The main idea is to insert $l$ vertices from $A_{1} \cup \ldots \cup A_{l}$ after every $k-l+1$ vertices in $H$ such that we get the $k$ th power of a Hamiltonian cycle. For this purpose, we define $B^{\prime}=\left\{b_{1}, b_{2}, \ldots, b_{\left\lfloor\frac{n}{k+1}\right\rfloor}\right\}$ in the following way: $b_{1}$ corresponds to the points $p_{1}, p_{2}, \ldots, p_{2(k-l+1)}, b_{2}$ corresponds to the points $p_{k-l+2}, p_{k-l+3}, \ldots, p_{3(k-l+1)}$, etc., and $b_{\left\lfloor\frac{n}{k+1}\right\rfloor}$ corresponds to the points $p_{\left(\left\lfloor\frac{n}{k+1}\right\rfloor-1\right)(k-l+1)+1}, \ldots, p_{|B|}, p_{1}, p_{2}, \ldots, p_{k-l+1}$. We 
also define $G^{\prime}$ on $A_{1} \cup \ldots \cup A_{l} \cup B^{\prime}$ as $\left.G\right|_{A_{1} \cup \ldots \cup A_{l}}$ and every $b_{i} \in B^{\prime}$ is connected to the common neighbors of all its corresponding points in $B$. Thus, in $G^{\prime}$, we have $\left|A_{1}\right|=\left|A_{2}\right|=\ldots=\left|A_{1}\right|=\left|B^{\prime}\right|=\left\lfloor\frac{n}{k+1}\right\rfloor$. At this point, it seems that all we have to do is to find a covering of $G^{\prime}$ by vertex-disjoint $(l+1)$-cliques by using Lemma 2.4 . However, if $l>1$, we require certain connections between the cliques of $b_{i}$ and $b_{i+1}$ in order to get the $k$ th power of a Hamiltonian cycle. To avoid this complication, we do the following. We cover every other $b_{i}$ with a $(l+1)$-clique in $G^{\prime}$ with the greedy procedure (for simplicity, we assume that $\left|B^{\prime}\right|$ is even, otherwise we cover three consecutive $b_{i}$ 's). Furthermore, in the process of this greedy procedure we always eliminate the arising exceptional vertices which do not have enough neighbors in the remaining part of one of the other sets in $A_{1}, \ldots, A_{l}, B^{\prime}$. These cliques provide an obvious restriction on the neighborhoods of the remaining $b_{i}$ 's. For example, if $l=2$, then the vertex in $A_{2}$ in the clique of $b_{i+1}$ must be connected to the vertex in $A_{1}$ in the clique of $b_{i}$. Taking into account these restrictions, we can find the cliques for the remaining $b_{i}$ 's by Lemma 2.4. We get the $k$ th power of a Hamiltonian cycle by inserting between $p_{i(k-l+1)}$ and $p_{i(k-l+1)+1}$ the other $l$ vertices in the clique of $b_{i}$ in the order $A_{l}, A_{l-1}, \ldots, A_{1}$.

In case $|B|>(k-l+1)\left\lfloor\frac{n}{k+1}\right\rfloor$, we do the following. As we construct the $(k-l)$ th power of a Hamiltonian cycle in $B$ with the method described in the previous sections, by utilizing the $(k-l+2)$-cliques, a short part of this Hamiltonian cycle is actually the $(k-l+1)$ st power of a path. Then on this part of the path we can contract $|B|-(k-$ $l+1)\left\lfloor\frac{n}{k+1}\right\rfloor$ edges into vertices so that the resulting graph still contains the $(k-l)$ th power of a Hamiltonian cycle. We do the above procedure for this Hamiltonian cycle and then by substituting the edges for the contracted vertices, we still get the $k$ th power of a Hamiltonian cycle in $G$.

The handling of the bad vertices is very similar to the above special extremal case and the details are left to the reader.

Finally, let $l=k-1$. We may also assume that there is a partition $B=B_{1} \cup B_{2}$ with $\left|B_{1}\right|=\left\lfloor\frac{n}{k+1}\right\rfloor$ and $d\left(B_{1}, B_{2}\right)<\alpha$, otherwise our method in [13] and the above inserting technique finishes the proof. Again for simplicity, we assume that there are no exceptional vertices. We find two vertex-disjoint edges $e_{1}=\left(u_{1}, v_{1}\right), e_{2}=\left(u_{2}, v_{2}\right)$ such that $u_{1}, u_{2} \in B_{1}$ and $v_{1}, v_{2} \in B_{2}$. We take a matching of size $\left|B_{2}\right|-\left\lfloor\frac{n}{k+1}\right\rfloor$ in $B_{2}$ that is vertex-disjoint from $e_{1}$ and $e_{2}$, and we collapse these edges into vertices. We still denote the resulting set by $B_{2}$, so $\left|B_{2}\right|=\left\lfloor\frac{n}{k+1}\right\rfloor$. We find Hamiltonian paths in $\left.G\right|_{B_{1}}$ connecting $u_{1}$ with $u_{2}$, and in $\left.G\right|_{B_{2}}$ connecting $v_{1}$ with $v_{2}$. Denote the resulting Hamiltonian cycle in $\left.G\right|_{B}$ by $H$. With the above inserting technique we insert $l$ vertices from $A_{1} \cup \ldots \cup A_{l}$ after every 2 vertices in $H$ such that we get the $k$ th power of a Hamiltonian cycle. This finishes the extremal case and the proof of Theorem 1.5.

\section{References}

1. B. Bollobás, Extremal Graph Theory, Academic Press, London, 1978.

2. G.A. Dirac, Some theorems on abstract graphs, Proc. London Math. Soc. 2 (1952) 68-81.

3. G. Fan and R. Häggkvist, The square of a hamiltonian cycle, SIAM J. Discrete Math., to appear.

4. G. Fan and H.A. Kierstead, The square of paths and cycles, manuscript. 
5. G. Fan and H.A. Kierstead, The square of paths and cycles, J, Combin. Theory Ser. B 63 (1995) 55-64.

6. G. Fan and H.A. Kierstead, Hamiltonian square-paths, J. Combin. Theory Ser. B 67 (1996) 167-182.

7. R.J. Faudree, R.J. Gould, and M. Jacobson, On a problem of Pósa and Seymour, private communication, 1992.

8. R.J. Faudree, R.J. Gould, M.S. Jacobson, and R.H. Schelp, On a problem of Paul Seymour, In: Recent Advances in Graph Theory, V.R. Kulli, Ed., Vishwa International Publication, 1991, pp. 197-215.

9. R. Häggkvist, On $F$-hamiltonian graphs, In: Graph Theory and Related Topics, J.A. Bondy and U.S.R. Murty, Eds,, Academic Press, New York, 1979, pp. 219-231.

10. A. Hajnal and E. Szemerédi, Proof of a conjecture of Erdős, In: Combinatorial Theory and Its Applications, Vol. II, P. Erdős, A. Rényi, and V.T. Sós, Eds., Colloq. Math. Soc. J. Bolyai 4, North-Holland, Amsterdam, 1970, pp. 601-623.

11. J. Komlós, G.N. Sárközy, and E. Szemerédi, Proof of a packing conjecture of Bollobás, Combin. Probab. Comput. 4 (1995) 241-255.

12. J. Komlós, G.N. Sárközy, and E. Szemerédi, Blow-up Lemma, Combinatorica 17 (1997) 109-123.

13. J. Komlós, G.N. Sárközy, and E. Szemerédi, On the square of a Hamiltonian cycle in dense graphs, Random Structures and Algorithms 9 (1996) 193-211.

14. J. Komlós, G.N. Sárközy, and E. Szemerédi, On the Pósa-Seymour conjecture, J. Graph Theory, to appear.

15. J. Komlós, G.N. Sárközy, and E. Szemerédi, An algorithmic version of the Blow-up Lemma, Random Structures and Algorithms, to appear.

16. P. Seymour, Problem section, In: Combinatorics: Proceedings of the British Combinatorial Conference 1973, T.P. McDonough and V.C. Mavron, Eds., Cambridge University Press, 1974, pp. 201-202.

17. E. Szemerédi, Regular partitions of graphs, In: Colloques Internationaux C.N.R.S. № 260 Problèmes Combinatoires et Théorie des Graphes, Orsay, 1976, pp. 399-401. 